\title{
Lebensqualität sticht bei Insektenallergie
}

\author{
Die Angst vor Insektenstichen bei bekannter Hymenopterengift- \\ allergie kann weit reichende emotionale und soziale Folgen haben. \\ Eine teilrandomisierte, kontrollierte Studie beleuchtet den Nutzen \\ einer spezifischen Immuntherapie (SIT) aus der Sicht der Patienten.
}

K ritiker der SIT für Patienten mit einer anaphylaktischen Insektenstichreaktion argumentieren, die Wahrscheinlichkeit eines erneuten Stiches sei zu gering, um den therapeutischen Aufwand zu rechtfertigen. Stattdessen empfehlen sie das Tragen eines Adrenalin-Selbstinjektionssets für den Notfall.

Allergologen der Universitätsklinik Groningen, Holland, verglichen beide Vorgehensweisen bezüglich ihres Effekts auf die selbst eingeschätzte Lebensqualität (LQ) der Betroffenen. Mit Hilfe eines Fragebogens erfassten sie die LQ der Patienten vor und nach einem Jahr mit SIT oder EpiPen. Alle Studienteilnehmer bekamen die gleiche Patienteninformation zu Nutzen und Risiko der Therapieoptionen. Von 69 Patienten, die einer Randomisierung zustimmten, erhielten 34 eine SIT (rSIT) und 35 den
EpiPen (rEPI). Weitere 66 Patienten lehnten eine Randomisierung ab und entschieden sich für die SIT (nr-SIT: $\mathrm{n}=$ 54) oder den EpiPen (nr-EPI: $\mathrm{n}=12$ ). Die Untersucher verzichteten auf Stichprovokationstests.

Während der Studie erlitten nur zwei Teilnehmer einen Bienen- oder Wespenstich. Nach einem Jahr war die mittlere LQ in beiden SIT-Gruppen wesentlich gestiegen $(p<0,0001)$, während die rEPI-Gruppe eine Verschlechterung ihrer LQ zeigte $(p<0,003)$. Die LQ der nr-EPI-Gruppe, also derjenigen, die sich bewusst für den EpiPen statt die SIT entschieden hatten, war nicht signifikant angestiegen. Die LQ verbesserte sich bei $74 \%$ der rSIT-Patienten, doch nur bei $9 \%$ der rEPI-Patienten. Sie verschlechterte sich bei $26 \%$ der rEPI-Patienten, aber bei keinem der rSIT-Patienten.

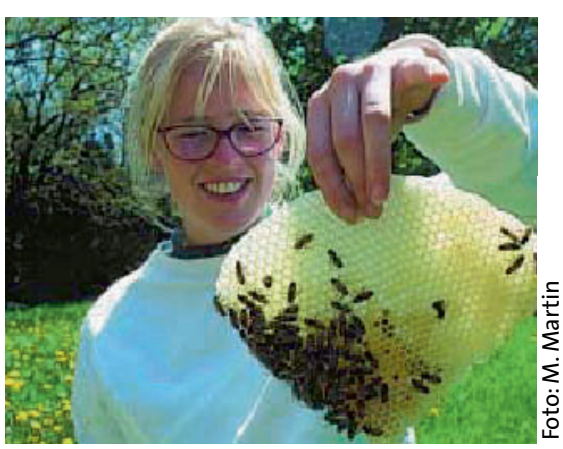

Eine Hyposensibiliserung sorgt bei zwei von drei Insektengiftallergikern für mehr Lebensqualität.

Mehr als 94\% der SIT-Patienten fanden, dass der Nutzen die Nachteile der Behandlung überwog.

Fazit: Unabhängig vom medizinischen Nutzen führt die SIT bei zwei von drei Patienten mit Hymenopterengiftallergie zur subjektiven Verbesserung der Lebensqualität.

Oude Elberink JN et al. Venom immunotherapy improves health-related quality of life in patients allergic to yellow jacket venom. J Allergy Clin Immunol 2002; 110: $174-82$

\section{Rhinitis und Asthma dieselbe Erkrankung?}

\section{Vor zwei Jahren hat die ARIA-Initiative (Allergic Rhinitis and its Impact on Asthma) ein neues Paradigma postuliert: Asthma und Rhinitis sind nur verschiedene Ausprägungen derselben Atemwegs- erkrankung. Die Resultate einer prospektiven, populationsbasierten Langzeitstudie könnten jetzt die letzten Skeptiker überzeugen.}

N ach neuesten Erkenntnissen ist die IgE-vermittelte Entzündung sowohl nasaler als auch bronchialer Schleimhäute, also allergische Rhinitis und allergisches Asthma, eine einheitliche Erkrankung. Doch fehlt trotz der attraktiven Hypothese bislang ein überzeugender epidemiologischer Nachweis. Vor diesem Hintergrund befragten dänische Forscher 734 Kopenhagener zweimalig (1990 und 1998) zu symptomatischen Reaktionen nach Allergenexposition. Zudem untersuchten sie die Probanden auf IgE-Antikör- per gegen Pollen, Tierepithelien oder Milben.

Von den Rhinitikern mit Pollenallergie reagierten im Jahr 1990 26\% (45 von 173) und im Jahr 1998 28\% (52 von 183) zugleich auch asthmatisch. Bei Tierallergikern war der Anteil asthmatischer Reaktionen bei allergischer Rhinitis sogar doppelt so hoch. Zu beiden Zeitpunkten litten 90-100\% der Asthmatiker zugleich auch unter allergischer Rhinitis. Bei der Zweituntersuchung hatten alle Asthmatiker mit Pollenallergie $(n=52)$ auch eine allergi- sche Rhinitis auf Pollen. Dasselbe galt für 31 der 35 Tierhaarallergiker und 19 der 20 Milbenallergiker. 48 Probanden entwickelten während des Studienzeitraums ein Asthma bronchiale, $47 \mathrm{da}$ von bei einer bereits bestehenden allergischen Rhinitis.

Fazit: Ein Viertel der Pollenrhinitiker und die Hälfte der Patienten mit allergischer Rhinitis gegen Tierhaare oder Milben zeigen bereits auch asthmatische Symptome. Andererseits hatten nahezu $100 \%$ derjenigen mit allergischem Asthma zusätzlich rhinitische Beschwerden. Der viel diskutierte $\mathrm{Zu}$ sammenhang beider Erkrankungen wird so erneut bestätigt.

Linneberg A et al. The link between allergic rhinitis and allergic asthma: a prospective population-based study. The Copenhagen Allergy Study. Allergy 2002; 57: $1048-52$ 